\title{
Lymphocyte activation gene 3 and coronary artery disease
}

\author{
Diana Golden, ${ }^{1}$ Antonina Kolmakova, ${ }^{1}$ Sunitha Sura, ${ }^{1}$ Anthony T. Vella, ${ }^{2}$ Ani Manichaikul, ${ }^{3,4}$ \\ Xin-Qun Wang, ${ }^{4}$ Suzette J. Bielinski, ${ }^{5}$ Kent D. Taylor, ${ }^{6}$ Yii-Der Ida Chen, ${ }^{6}$ Stephen S. Rich, ${ }^{3}$ \\ and Annabelle Rodriguez ${ }^{1}$ \\ 1 Center for Vascular Biology and ${ }^{2}$ Department of Immunology, University of Connecticut Health Center, Farmington, \\ Connecticut, USA. ${ }^{3}$ Center for Public Health Genomics, University of Virginia, Charlottesville, Virginia. ${ }^{4}$ Biostatistics Section, \\ Department of Public Health Sciences, University of Virginia, Charlottesville, Virginia, USA. ${ }^{5}$ Division of Epidemiology, \\ Mayo Clinic, Rochester, Minnesota, USA. 'os Angeles BioMedical Research Institute at Harbor-UCLA Medical Center, \\ Los Angeles, California, USA.
}

BACKGROUND: The lipoprotein scavenger receptor BI (SCARB1) rs10846744 noncoding variant is significantly associated with atherosclerotic disease independently of traditional cardiovascular risk factors. We identified a potentially novel connection between rs10846744, the immune checkpoint inhibitor lymphocyte activation gene 3 (LAC3), and atherosclerosis.

METHODS: In vitro approaches included flow cytometry, lipid raft isolation, phosphosignaling, cytokine measurements, and overexpressing and silencing LAG3 protein. Fasting plasma LAG3 protein was measured in hyperalphalipoproteinemic (HALP) and Multi-Ethnic Study of Atherosclerosis (MESA) participants.

RESULTS: In comparison with rs10846744 reference (GC homozygous) cells, LAC3 protein levels by flow cytometry $(P<0.001)$, in lipid rafts stimulated and unstimulated $(P=0.03)$, and phosphosignaling downstream of $B$ cell receptor engagement of CD79A $(P=0.04), \operatorname{CD19}(P=$ 0.04), and LYN $(P=0.001$ ) were lower in rs10846744 risk (CC homozygous) cells. Overexpressing LAC3 protein in risk cells and silencing LAG3 in reference cells confirmed its importance in phosphosignaling. Secretion of TNF- $\alpha$ was higher $(P=0.04)$ and IL-10 was lower $(P=0.04)$ in risk cells. Plasma LAC3 levels were lower in HALP carriers of the CC allele $(P<0.0001)$ and by race $(P=0.004)$. In MESA, race $(P=0.0005)$, age $(P=0.003)$, lipid medications $(P=$ $0.03)$, smoking history $(P<0.0001)$, and rs10846744 genotype $(P=0.002)$ were independent predictors of plasma LAG3. In multivariable regression models, plasma LAC3 was significantly associated with HDL-cholesterol (HDL-C) $(P=0.007)$, plasma IL-10 $(P<0.0001)$, and provided additional predictive value above the Framingham risk score $(P=0.04)$. In MESA, when stratified by high HDL-C, plasma LAC3 was associated with coronary heart disease (CHD) (odds ratio $1.45, P=0.004)$.

CONCLUSION: Plasma LAG3 is a potentially novel independent predictor of HDL-C levels and CHD risk.

FUNDING: This work was supported by an NIH R01 grant (HL075646), the endowed Linda and David Roth Chair for Cardiovascular Research, and the Harold S. Geneen Charitable Trust Coronary Heart Disease Research award to Annabelle Rodriguez. MESA is conducted and supported by the National Heart, Lung, and Blood Institute (NHLBI) in collaboration with MESA

Conflict of interest: A.R. has patent rights to materials related to the manuscript and she is the founder of Lipid Genomics.

Submitted: May 24, 2016 Accepted: September 19, 2016 Published: October 20, 2016

Reference information: JCI Insight. 2016;1(17):e88628 doi:10.1172/jci.insight.88628. investigators. Support for MESA is provided by contracts HHSN268201500003I, N01-HC-95159, N01-HC-95160, N01-HC-95161, N01-HC-95162, N01-HC-95163, N01-HC-95164, N01-HC-95165, N01-HC-95166, N01-HC-95167, N01-HC-95168, N01-HC-95169, UL1-TR-001079, UL1-TR-000040, and DK063491. Cardiometabochip genotyping data for the MESA samples was supported in part by grants and contracts R01HL98077, N02-HL-64278, HL071205, UL1TR000124, DK063491, RD831697, and P50 ES015915. 


\section{Introduction}

The lipoprotein receptor, scavenger receptor class B type I (SR-BI), is a physiologically relevant receptor that modulates cholesterol levels, especially HDL-cholesterol (HDL-C), in mice and humans (1-7). We previously showed that the rs10846744 SNP within the SR-BI gene, SCARB1 (12q24.31), was significantly associated with subclinical atherosclerosis (SCA), myocardial infarction (MI), and cardiovascular disease (CVD) in male participants of the Multi-Ethnic Study of Atherosclerosis (MESA) $(5,6)$. Specifically, homozygous carriers of the rs 10846744 risk genotype (CC) had significantly increased odds for MI and CVD, and in a multivariable regression model this association was not attenuated by inclusion of traditional CVD risk factors such as age, BMI, hypertension, smoking, renal disease, lipid-lowering medications including statin use, or lipid levels (whether total cholesterol [TC], LDL-cholesterol [LDL-C], HDL-C, or triglycerides [TGs]). These findings strongly suggested that other factors or pathways might be causal in the association of rs10846744 with CVD.

The rs10846744 SNP resides within the first intron of SCARB1 and bioinformatic analysis revealed that this SNP is located within an enhancer region, suggesting a region that could transcriptionally regulate genes intrachromosomally or interchromosomally (8). RNA-Seq was used to evaluate differentially expressed transcripts from lymphocytes isolated from homozygous reference $(G)$ or risk $(C)$ allele carriers. A number of transcriptionally regulated gene candidates emerged, including lymphocyte activation gene 3 (LAG3) that is located at $12 \mathrm{p} 13.32$.

LAG3 is an Ig superfamily member protein and binds MHC class II with high affinity, and is a potent immune checkpoint inhibitor of activated $\mathrm{T}$ cells $(9,10)$. It is expressed in $\mathrm{B}, \mathrm{T}$, and NK cells, monocytes, and dendritic cells (11), and its major function is to suppress activated $\mathrm{T}$ cells by controlling effector $\mathrm{T}$ cell expansion and homeostasis (12). Cell surface LAG3 protein is cleaved by ADAM10 and ADAM17 metalloproteases, generating plasma LAG3 (13). In vitro and in vivo murine studies have suggested that plasma can also limit $\mathrm{T}$ cell activation and homeostasis by binding to MHC class II on antigen-presenting cells (13). Importantly, a few clinical studies have shown associations between plasma LAG3 and tuberculosis resistance (14) and breast cancer prognosis (15).

Since previous analyses showed that traditional CVD risk factors did not attenuate the association of rs10846744 with SCA and CVD $(5,6)$, we hypothesized that LAG3 protein might affect atherosclerotic risk. Therefore, we used a number of approaches to examine the expression of LAG3 in EBV-transformed $B$ lymphocytes as well as its association with clinical outcomes such as coronary heart disease (CHD). We observed that plasma LAG3 was an independent predictor of HDL-C and CHD risk in the large MESA.

\section{Results}

Study demographics. The overall study design, inclusion and exclusion criteria, biospecimen acquisition, and clinical morbidity and mortality for the HALP and MESA cohorts is shown in Figure 1. As shown in Table 1, HALP subjects were, on average, middle-aged and predominantly white females. At the time of enrollment none of the subjects were treated with cholesterol-lowering medications whether prescribed or over the counter. The MESA cohort was older and approximately equally distributed between sexes with a larger percentage of patients with Mixed European Descent (40\%) compared with African-Americans (26\%), Hispanics (22\%), and Asians (12\%). Since HALP subjects were a priori recruited for fasting plasma HDL-C greater than or equal to $60 \mathrm{mg} / \mathrm{dl}$, the mean HDL-C was $83 \mathrm{mg} / \mathrm{dl}$ as compared with the MESA cohort (mean HDL-C $52 \mathrm{mg} / \mathrm{dl}$ ), since recruitment for this study was based upon age and participants being free from clinical CVD at baseline. Plasma LAG3 levels were significantly different between $\operatorname{HALP}(8,983 \pm 893 \mathrm{pg} / \mathrm{ml})$ and MESA subjects $(1,867 \pm 54)$ $(P<0.0001)$. In MESA participants, the minor allele frequency of rs 10846744 differed significantly between patients of Mixed European Descent with Chinese-Americans $(P<0.0001)$ and with AfricanAmericans $(P<0.0001)$, but not with Hispanics (Supplemental Figure 1; supplemental material available online with this article; doi:10.1172/jci.insight.88628DS1).

Transcriptome analysis reveals differential expression of $L A G 3 R N A$. We first examined transcriptional differences between the homozygous reference (GG homozygous) and risk (CC homozygous) cells cultured under basal (unstimulated) conditions. We explored transcriptional differences of targets residing on chromosome 12, and identified 5 gene transcripts that were significantly downregulated and 3 gene transcripts upregulated in risk cells as compared with the reference cells (Supplemental Table 1). Using real-time PCR and Western blotting, we verified that $L A G 3$ RNA and LAG3 protein expression were lower $(P=0.001$ 


\section{Hyperalphalipoproteinemic (HALP) Observational Cohort}

HALP is a single-site ongoing study recruiting community dwelling adults between the ages of $18-80$ years. The study began in April 2004 at the Johns Hopkins University School of Medicine and then the study was transferred to the University of Connecticut

Health School of Medicine in Sept 2012. Dr. Rodriguez is the Principal Investigator of this study and has been since its inception.

The rationale for the HALP study was to identify subjects with fasting HDL-cholesterol (HDL-C) levels $\geq 60 \mathrm{mg} / \mathrm{dl}$ with SCARB1 single nucleotide polymorphisms, insertions, and/or deletions that were significantly associated with low SR-BI macrophage protein levels. This is NOT an interventional study.

Inclusion criteria: fasting HDL-C $\geq 60 \mathrm{mg} / \mathrm{dl}$; normal thyroid, kidney, liver function tests, and negative urine pregnancy test for women of child-bearing age.

Exclusion criteria: fasting HDL Inclusion criteria: fasting HDL-C $<60 \mathrm{mg} / \mathrm{dl}$; normal thyroid, kidney, liver function tests and/or positive urine pregnancy test in women of child-bearing age.

Enrolled subjects donated fasting blood for isolation of DNA for SCARB1 genotyping, lipid and lipoprotein measurements, general chemistry panel, plasma and serum storage for future studies, and peripheral blood mononuclear storage.

Completed enrollment: $\mathrm{N}=159(\mathrm{~N}=106$ Hopkins, $\mathrm{N}=53$ UConn)

Disqualified: $\mathrm{N}=47$, reasons included $\mathrm{HDL}<60 \mathrm{mg} / \mathrm{dl}$, abnormal thyroid or general chemistry lab results

Plasma samples available for testing for LAG3: $\mathrm{N}=151$

Missing plasma samples: $\mathrm{N}=8$ aliquots per subject were not available

\section{Multi-Ethnic Study of Atherosclerosis (MESA) Observational Cohort}

MESA is a multi-site ongoing study recruiting community dwelling adults between the ages of $45-84$ years, which began in July 2000. The six field study sites include Johns Hopkins University School of Medicine, Baltimore, MD; Wake Forest School of Medicine, Winston-Salem, NC; Columbia University Medical Center, New York, NY; University of Minnesota, Minneapolis, MN; Northwestern University, Chicago, IL; Harbor-UCLA School of Medicine, Los Angeles, CA.

The primary objectives for the MESA study were to determine characteristics related to progression of subclinical to clinical cardiovascular disease (CVD) and to determine characteristics related to progression of subclinical CVD in subjects from four ethnic groups: Mixed European Descent, African-Americans, Hispanics, and Chinese-Americans. Study visits occurred every 2 years. Final enrollment: $\mathrm{N}=6814$ men and women. This is NOT an interventional study.

Inclusion criteria: Free of CVD at baseline; ages between $45-84$ years.

Exclusion criteria: Subjects with known CVD; age cutoffs below 45 and above 84 years; weight $>300$ lbs; living in a nursing home; chest CT scan in past year of enrollment; language barrier; cognitive inability; pregnancy, active treatment in cancer

Enrolled subjects donated fasting blood for isolation of DNA for genotyping, lipid and lipoprotein measurements, general chemistry panel, and plasma and serum storage for future studies. Study subjects were contacted every 9 -

12 months to assess clinical morbidity and mortality. Study subjects provided written consent for cardiovascular imaging studies including carotid ultrasound and coronary artery calcium scan.

\begin{tabular}{|c|}
\hline Completed enrollment at visit 2: $\mathrm{N}=6429$ \\
\hline $\begin{array}{c}\text { Plasma samples available for testing for LAG3 from visit } 2 \text { samples: } \mathrm{N}=5623 \\
\text { Missing plasma samples: } \mathrm{N}=806 \text { aliquots per subject were not available }\end{array}$ \\
\hline $\begin{array}{c}\text { Clinical morbidity and mortality data obtained from visit } 2 \text { through visit } 5(2013): \\
\text { Coronary heart disease and mortality } \mathrm{N}=5618, \text { missing data: } \mathrm{N}=5 \text {. } \\
\text { Carotid intimal medial thickness }(\mathrm{N}=4973) \text { and coronary artery calcium scan }(\mathrm{N}=2440)\end{array}$ \\
\hline
\end{tabular}

Figure 1. Overall study design for the HALP and MESA cohorts. 
Table 1. Study demographics of HALP and MESA population

\begin{tabular}{|c|c|c|}
\hline & HALP & MESA \\
\hline$n$ & 150 & 5,623 \\
\hline Age (yrs, mean \pm SD, [range]) & $52.9 \pm 13.0[18-81]$ & $63.7 \pm 10.1[46-87]$ \\
\hline \multicolumn{3}{|l|}{ Race $(n, \%)$} \\
\hline Mixed European Descent & $107(74 \%)$ & $2,382(40 \%)$ \\
\hline African-American & $24(17 \%)$ & $1,503(26 \%)$ \\
\hline Asian & $7(5 \%)$ & $706(12 \%)$ \\
\hline Hispanic & $6(4 \%)$ & $1,309(22 \%)$ \\
\hline \multicolumn{3}{|l|}{ Gender ( $n, \%)$} \\
\hline Male & $27(19 \%)$ & $3,060(52 \%)$ \\
\hline Female & $118(81 \%)$ & $2,840(48 \%)$ \\
\hline Total cholesterol ${ }^{A}(\mathrm{mg} / \mathrm{dl} \pm \mathrm{SD}$, [range]) & $213 \pm 37[123-295]$ & $191 \pm 36[52-460]$ \\
\hline Triglycerides (mg/dl) & $75 \pm 31[27-209]$ & $132 \pm 82[24-1,128]$ \\
\hline LDL-C (mg/dl) & $114 \pm 31[49-195]$ & $114 \pm 32[18-377]$ \\
\hline HDL-C (mg/dl) & $83 \pm 20[60-156]$ & $52 \pm 15[21-161]$ \\
\hline Plasma LAG3 (pg/ml, mean \pm SEM, [range]) & $8,983 \pm 893[0-58,646]$ & $1,867 \pm 54[0-51,203]$ \\
\hline \multicolumn{3}{|l|}{ SCARB1 rs10846744 ( $n, \%)$} \\
\hline GG & $105(69 \%)$ & $2,448(44 \%)$ \\
\hline GC & $24(16 \%)$ & 2,186 (39\%) \\
\hline CC & 22 (15\%) & 974 (17\%) \\
\hline
\end{tabular}

ATo convert total cholesterol, LDL-cholesterol (LDL-C), and HDL-C from $\mathrm{mg} / \mathrm{dl}$ to $\mathrm{mmol} / \mathrm{l}$ multiply values by 0.02586 . To convert triglycerides from $\mathrm{mg} / \mathrm{dl}$ to $\mathrm{mmol} / \mathrm{I}$ multiple values by 0.01129 . CC, homozygous carriers of the rs 10846744 risk genotype; GG, homozygous carriers of the rs10846744 reference genotype; CC, heterozygous carriers.

and 0.05 , respectively) in risk cells as compared with reference cells (Supplemental Figure 2). In addition to transcriptome differences on chromosome 12, we also observed interchromosomal transcriptional differences (Supplemental Table 2; the full RNA-Seq data set is available in the NCBI's Gene Expression Omnibus [GEO GSE87891]).

LAG3 protein expression is significantly lower in rs 10846744 risk cells. At baseline, LAG3 cell surface protein expression was $90 \%$ lower in the risk cells as compared with reference cells $(P<0.001)$ (Figure 2, A-C). Following stimulation with PMA/ionomycin+IL-4, as compared with baseline levels, LAG3 cell surface protein expression decreased over time in both reference $(P<0.001)$ and risk cells (combined $P=0.04$ ) (Figure $2 \mathrm{C}$ ). In parallel, as compared with baseline levels, LAG3 protein levels increased over time in the medium from the reference cells $(P=0.03)$ as compared with no changes observed in the risk cells (Figure 2D). With stimulation, cytokine levels in the medium (Figure 3A) increased over time as compared with basal levels in both reference and risk cells (TNF- $\alpha, P<0.001$ for reference and risk cells, respectively; IL-10, $P=0.02$ for reference cells and $P<0.001$ for risk cells). TNF- $\alpha$ levels were significantly higher in risk cells (4-fold higher, $P=0.04$ ), while IL-10 levels were lower (53\% lower, $P=0.04$ ) as compared with reference cells (Figure 3B).

LAG3 protein expression is reduced in lipid rafts and downstream signaling is impaired in rs 10846744 risk (CC) cells. Since LAG3 protein has been shown to localize in lipid rafts in activated cells (16), we examined LAG3 protein localization in lipid rafts isolated from unstimulated and stimulated reference and risk cells. LAG3 expression in lipid rafts was lower in risk cells as compared with reference cells $(P=0.03)$ (Supplemental Figure 3). We tested whether LAG3 protein reduction affected downstream signaling pathways. Total B cell receptor (BCR) signaling proteins were detected in all cell lines in the absence of stimulation (Figure 4A). Following stimulation, none of the phosphorylated targets were detected in the risk cells, while all phosphorylated targets were expressed in the reference cells compared with the unstimulated condition p-CD79A $(P=0.04)$, p-CD19 $(P=0.04)$, p-SYK $(P=0.005)$, p-LYN $(P=0.001)$, p-PLC $\gamma 2(P=0.004)$ and p-PKC $\beta(P=0.003)$. Similar findings were observed of BCR phosphosignaling in all 3 independent reference and all 3 independent risk cells (Supplemental Figure 4). 
A

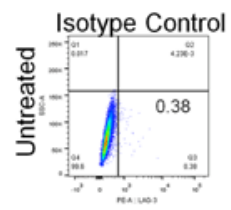

Reference (GG) expressing cells: Cell line 003
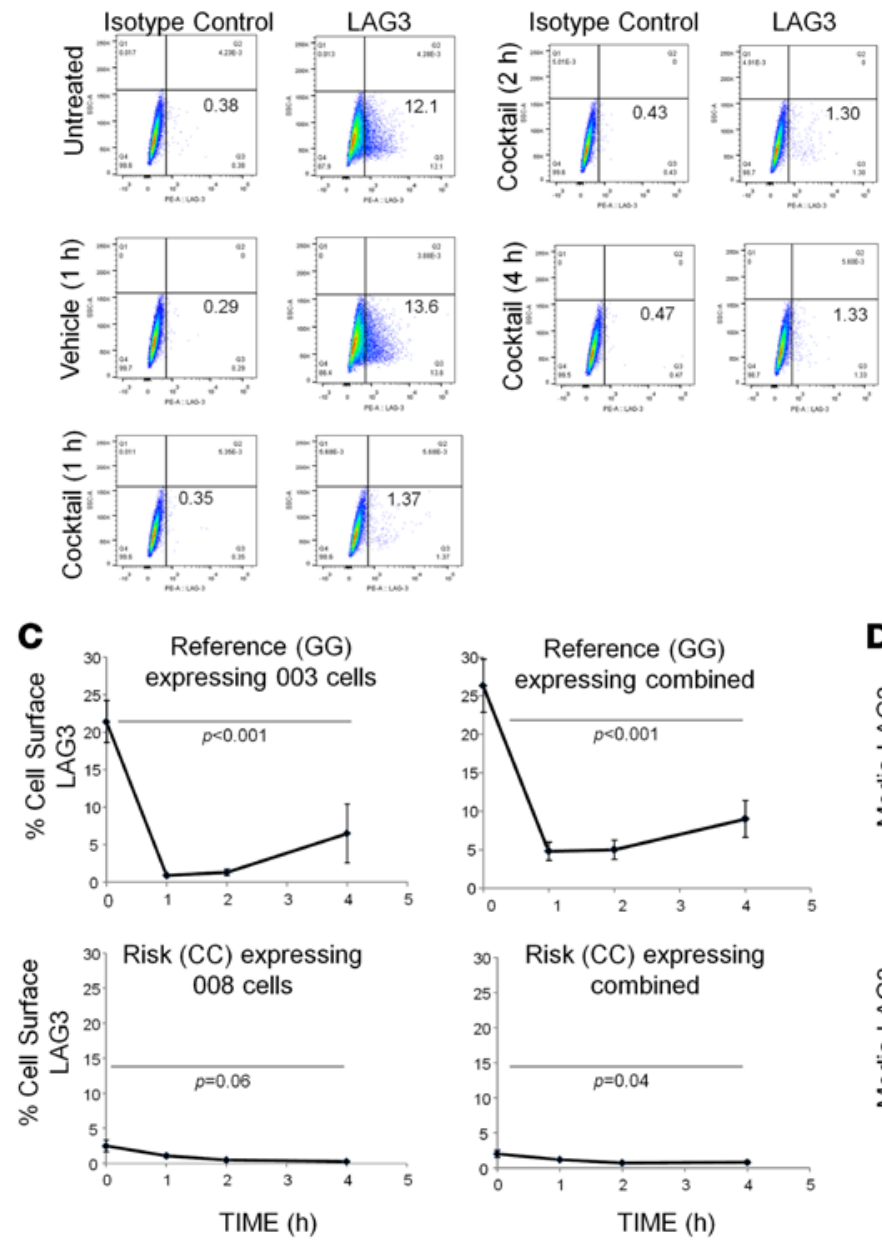

B Risk (CC) expressing cells: Cell line 008
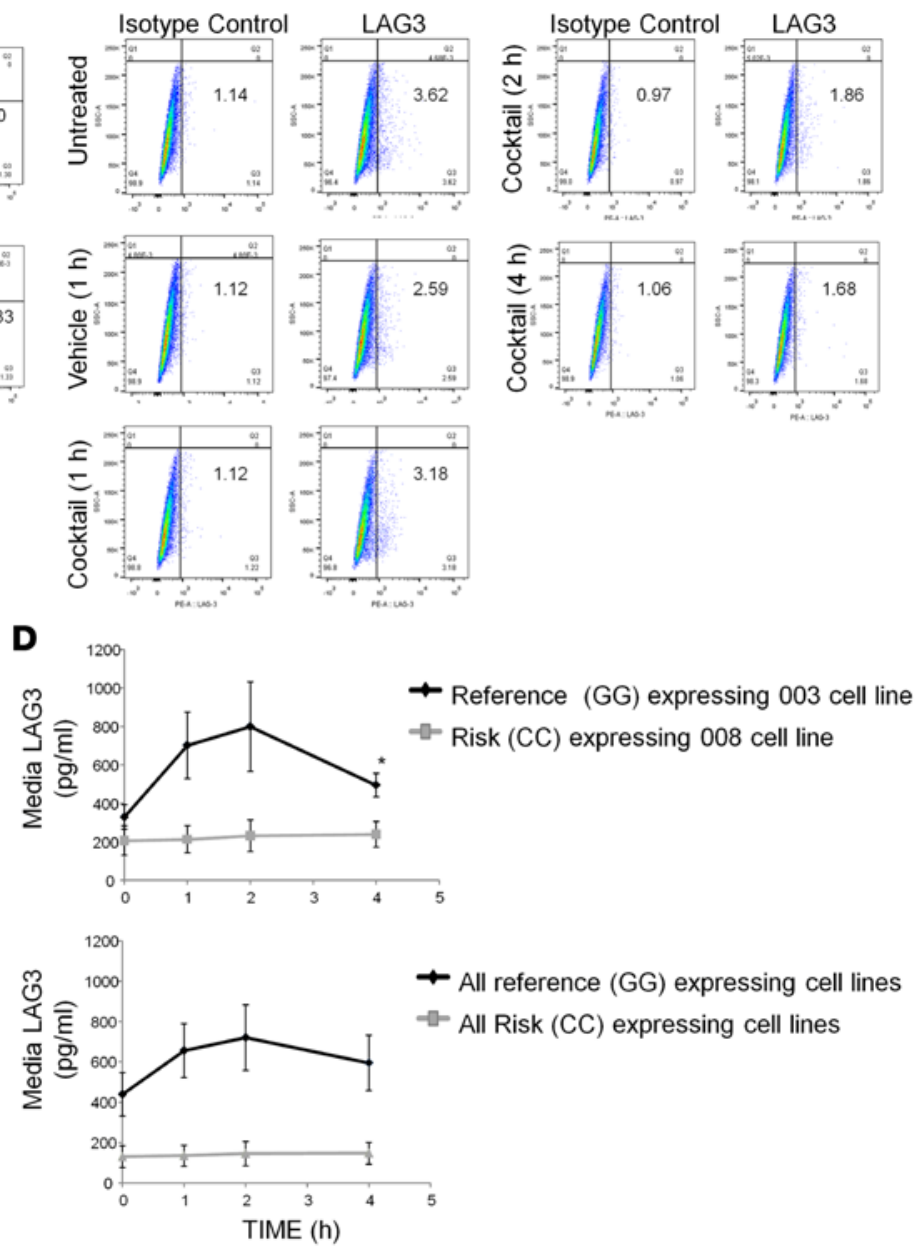

$\leftarrow$ All reference (GG) expressing cell lines - All Risk (CC) expressing cell lines

Figure 2. Differential expression of LAG3 protein and cytokine effects in SCARB1 rs10846744 reference (GG) and risk (CC) cells. EBV-transformed B lymphocytes homozygous for the reference (CC) or risk (CC) allele were incubated under basal or stimulated cocktail (500 ng/ml PMA, $250 \mathrm{ng} / \mathrm{ml}$ ionomycin, and $100 \mathrm{U} / \mathrm{ml} \mathrm{IL-4)}$ conditions for 0-4 hours and stained with isotype control or anti-human LAC3-PE monoclonal antibody for measurement of percentage cell surface LAG3 protein, and then fixed for flow cytometry. (A) Reference cells (003 cell line) under basal and stimulated conditions were stained with isotype control or LAG3 antibodies; the data are representative of 1 of 3 independent experiments. (B) Risk cells (008 cell line) under basal and stimulated conditions were stained with isotype control or anti-human LAG3-PE monoclonal antibody; the data are representative of 1 of 3 independent experiments. (C) Graphic analysis of percentage cell surface protein expression of LAG3+ cells as measured by flow cytometry. Top left panel represents pooled data (mean \pm SEM) of 3 independent experiments from the reference (GG-003) cells, each experiment performed with triplicate samples ( $n=9, P<$ 0.001 compared with baseline). Top right panel represents pooled data (mean \pm SEM) from 3 independent reference cell lines $(n=18, P<0.001$ compared with baseline). Bottom left presents pooled data (mean \pm SEM) of 3 independent experiments from the risk (CC-008) cells, each experiment performed with triplicate samples $(n=9, P=0.06$ ). Bottom right panel represents pooled data (mean \pm SEM) from all the risk cell lines $(n=15, P=0.04)$. (D) Changes in LAG3 protein levels in the media over time following cocktail (PMA/ionomycin+IL-4) stimulation in reference-expressing and risk-expressing cells. Top panel represents pooled data (mean \pm SEM) of 3 independent experiments from the reference (GG-003) cell line, each experiment performed with duplicate samples ( $n=6,{ }^{*} P=0.03$ compared with baseline), and from pooled data (mean \pm SEM) of 3 independent experiments from the risk (CC-008) cell line, each experiment performed with duplicate samples $(n=6, P \geq 0.05$, not significant). Bottom panel represents pooled data (mean \pm SEM) from all the reference cell lines $(n=12, P \geq 0.05$, not significant) and pooled data (mean \pm SEM) from all the risk cell lines $(n=10, P \geq 0.05$, not significant). Quadratic polynomial statistical analyses were performed to test significance.

Overexpression or silencing of LAG3 protein impacts downstream signaling pathways. To directly assess the effect of LAG3 protein on phosphosignaling, we performed experiments wherein LAG3 was (a) overexpressed in risk cells or (b) silenced in reference cells. LAG3 overexpression in basal or stimulated risk cells was associated with increased levels of phosphorylated targets $(P=0.04$ for $\mathrm{p}-\mathrm{LYN} ; P=0.03$ for $\mathrm{p}$-PKC $\beta$ in unstimulated cells and $P=0.01$ for $\mathrm{p}-\mathrm{LYN} ; P=0.01$ for $\mathrm{p}-\mathrm{PKC} \beta$ in stimulated cells) as compared with control cells (these being cells that were transfected with empty vector) (Figure 4B). LAG3 protein silencing was associated with lower levels of phosphorylated targets ( $P=0.002$ for $\mathrm{p}-\mathrm{LYN} ; P=0.009$ for $\mathrm{p}-\mathrm{PKC} \beta$ in stimulated cells) in the reference cells as compared with control cells (Figure $4 \mathrm{C}$ ). We stimulated overex- 

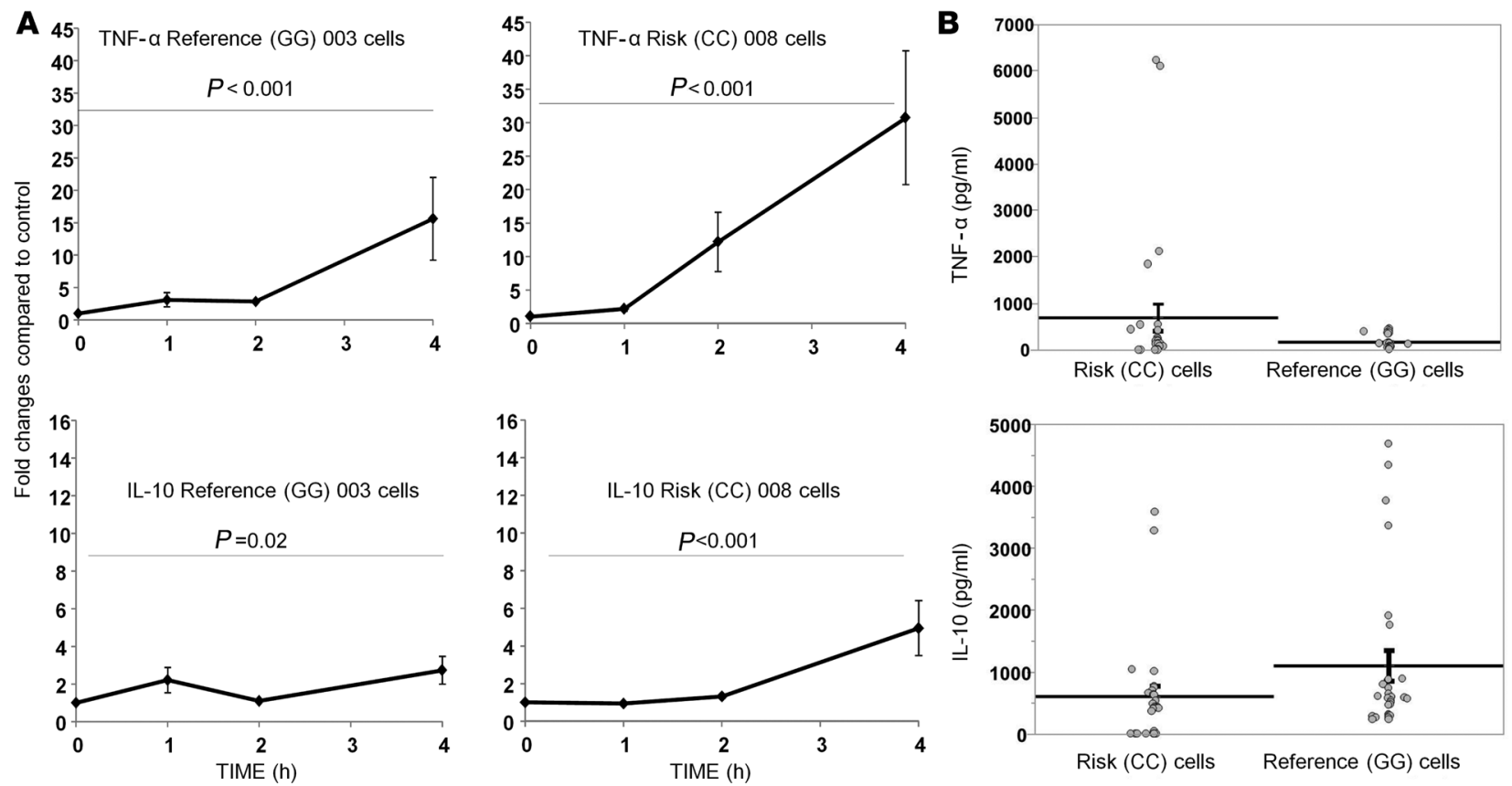

Figure 3. Changes in secreted cytokine (TNF- $\alpha$ and IL-10) levels in the media over time following activation in reference-expressing (GG-003) and riskexpressing (CC-008) cells. (A) Top left panel represents TNF- $\alpha$ pooled data (mean \pm SEM) of 3 independent experiments from the reference cell line, each experiment performed with duplicate samples $(n=6, P<0.001$ compared with baseline), while top right panel represents TNF- $\alpha$ pooled data (mean \pm SEM) of 3 independent experiments from the risk cell line, each experiment performed with duplicate samples $(n=6, P<0.001$ compared with baseline). Bottom left panel represents IL-10 pooled data (mean \pm SEM) from the reference cell line, each experiment performed with duplicate samples $(n=6, P=0.02$ compared with baseline), while bottom right panel represents IL-10 pooled data (mean \pm SEM) of 3 independent experiments from the risk cell line, each experiment performed with duplicate samples $(n=6, P<0.001)$. (B) Secretion of TNF- $\alpha$ and IL-10 from reference-expressing and risk-expressing cells. The results shown are mean \pm SEM of data from pooling all time points from each individual group. TNF- $\alpha$ levels were significantly higher in risk cells as compared with reference cells $(P=0.04)$. IL-10 levels were significantly higher in reference cells as compared with risk cells $(P=0.04)$. Quadratic polynomial regression models were used in analyses in (A); 2-sided Student's $t$ test was used for 2-sample analysis in B. A $P$ value less than 0.05 was considered significant.

pressing LAG3 risk cells separately with PMA/ionomycin+IL-4 or CD40 ligand and found that p-CD79A and $\mathrm{p}$-PKC $\beta$, which are specific to BCR-stimulated signaling and not $\mathrm{CD} 40 \mathrm{~L}$ signaling, were not activated in mock vector risk cells, but were now activated in cells overexpressing the LAG3-GFP construct (Supplemental Figures 5 and 6).

Homozygous HALP carriers of the rs10846744 risk allele (CC) had significantly lower plasma LAG3 protein levels. We determined if LAG3 protein levels would be significantly different in plasma isolated from HALP participants. In binary analysis, plasma LAG3 protein levels were significantly lower in carriers homozygous for the risk allele ( $n=22$; median [first quartile; third quartile]: $1,949[380 ; 5,468] \mathrm{pg} / \mathrm{ml} ; P<0.0001)$ as compared with subjects homozygous for the reference allele $(n=103 ; 6,907[3,399 ; 10,794])$ or heterozygous subjects (GC) $(n=24 ; 6,902[3,787 ; 10,927])$ (Figure 5 ; plasma LAG3 shown as log-transformed). We further analyzed the association of race/ethnicity with plasma LAG3 levels, and found that plasma LAG3 levels were significantly lower in African-Americans $(n=22 ; 2,772[380 ; 7,336] \mathrm{pg} / \mathrm{ml} ; P=0.004)$ compared with those of Mixed European Descent ( $n=101 ; 6,184[3,437 ; 10,809])$, Asians $(n=7 ; 9,602[3,273 ; 20,078])$, or Hispanics $(n=6 ; 7,723[4,105 ; 12,767])$ (Figure 6; plasma LAG3 shown as log-transformed). Multivariable linear regression analysis with adjustment for age, sex, and race/ethnicity showed that race $(P=0.009)$ and SCARBI rs10846744 $(P<0.0001)$ remained as significant independent predictors of plasma LAG3 levels.

Race/ethnicity, SCARB1 rs10846744 and other covariates are independent predictors of plasma LAG3 levels in $M E S A$. In binary analysis, plasma LAG3 protein levels were significantly higher in carriers homozygous for the risk allele ( $n=974$; median [first quartile; third quartile]: $610[240 ; 1,892] \mathrm{pg} / \mathrm{ml} ; P<0.0001)$ as compared with subjects homozygous for the reference allele $(n=2,448 ; 454[216 ; 1,252] \mathrm{pg} / \mathrm{ml})$ or heterozygous subjects (GC, $n=2,185 ; 485[251 ; 1,130] \mathrm{pg} / \mathrm{ml}$ ) (Figure 7, plasma LAG3 shown as log-transformed). We analyzed the association of race/ethnicity with plasma LAG3 levels, and found that plasma LAG3 levels were significantly higher in African-Americans $(n=1,428,507[202 ; 1,971] \mathrm{pg} / \mathrm{ml})$ and Chinese-Americans 


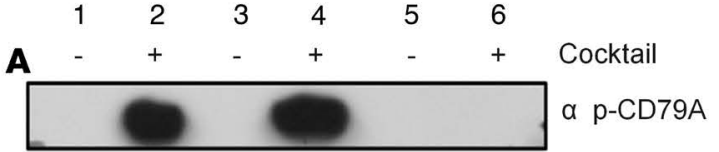

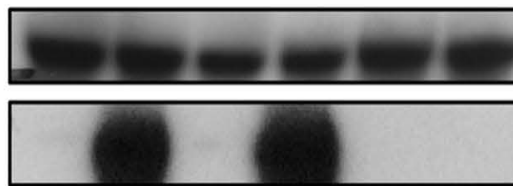

a CD79A

a p-CD19
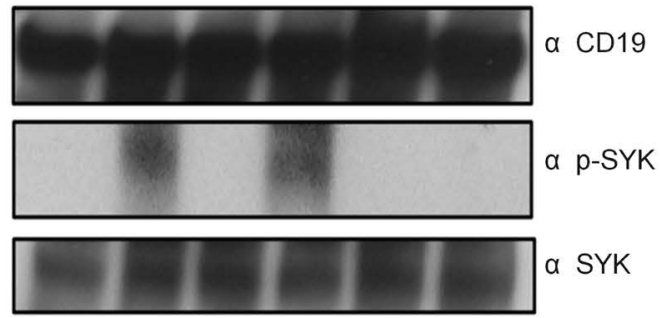

a SYK
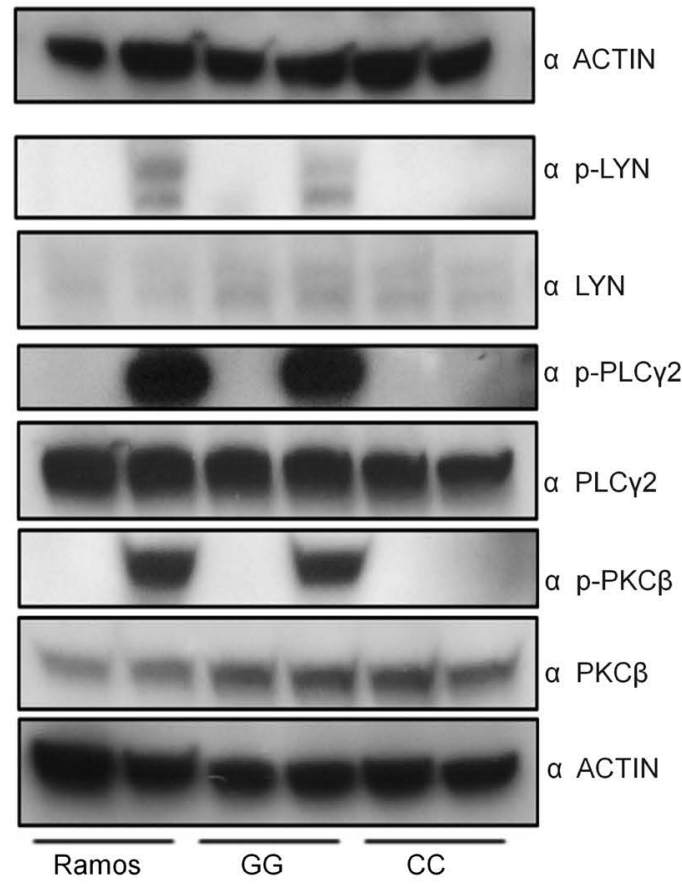
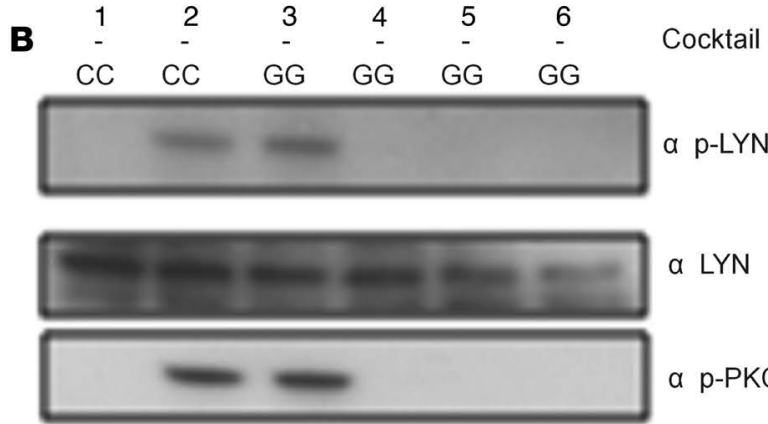

a LYN

$\alpha \mathrm{p}-\mathrm{PKC} \beta$

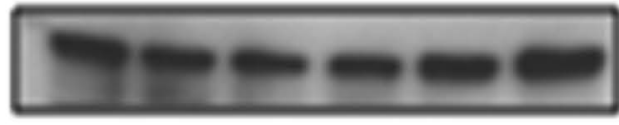

$\alpha \operatorname{PKC} \beta$
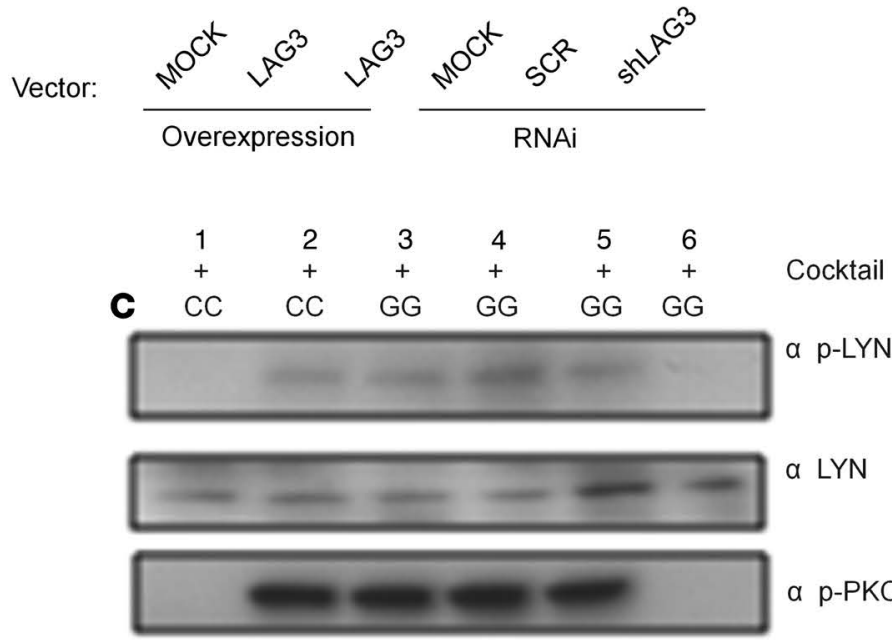

a LYN

$\alpha$ p-PKC $\beta$

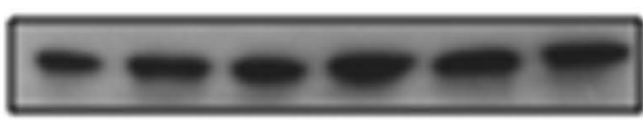

$\alpha \mathrm{PKC} \beta$

Vector:

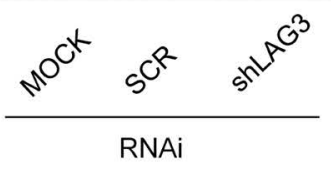

Overexpression

RNAi

Figure 4. LAG3 protein is crucial in B cell receptor (BCR) signaling. Protein was isolated from EBV-transformed B cells expressing the reference (CG) or risk (CC) allele under basal or cocktail-stimulated (PMA/ionomycin+IL-4) conditions for 2 hours. (A) Ramos cell line as control B cells, reference (GG-003) cell line or risk (CC-008) cell line. As compared with basal conditions, p-CD79A $(P=0.04), \mathrm{p}$-CD19 $(P=0.04), \mathrm{p}-\mathrm{SYK}(P=0.005), \mathrm{p}-\mathrm{LYN}(P=0.001), \mathrm{p}-\mathrm{PLC} \gamma 2$ $(P=0.004)$ and $p-\operatorname{PKC~} \beta(P=0.003)$ were increased in stimulated reference cells. Similar experiments were performed for additional cell lines derived from subjects expressing the reference or risk allele for a total of 6 independent cell lines $(n=3$ for the reference cells and $n=3$ for the risk-expressing cells) (Supplemental Figure 4). (B) The effect of overexpression of lentiviral LAG3-GFP or short-hairpin, shRNA-LAG3 silencing on BCR phosphosignaling in unstimulated cells. Lanes: 1, mock transduction in risk cells; 2, transduction of lentiviral LAG3-GFP in risk cells; 3, transduction of lentiviral LAG3-GFP in reference cells; 4 , mock transduction in reference cells; 5 , transduction of scrambled shRNA in reference cells; and 6, transduction of shRNA-LAC3 in reference cells. As compared with cells transduced with the mock vector, $\mathrm{p}$-LYN $(P=0.04)$ and $\mathrm{p}$-PKC $\beta(P=0.03)$ were increased in risk cells overexpressing LAC3 protein (lane 2), while p-LYN $(P=0.04)$ and $\mathrm{p}-\mathrm{PKC} \beta(P=0.01)$ were increased in reference cells overexpressing LAC3 protein (lane 3$)$ as compared with cells expressing the mock vector. (C) The effect of overexpression of lentiviral LAC3-GFP or shRNA-LAC3 silencing on BCR phosphosignaling in stimulated cells. Lanes: 1, mock transduction in risk cells; 2, transduction of lentiviral LAC3-GFP in risk cells; 3, transduction of lentiviral LAG3-GFP in reference cells; 4 , mock transduction in reference cells; 5 , transduction of scrambled shRNA in reference cells; and 6 , transduction of shRNA-LAG3 in reference cells. As compared with cells transduced with mock vector, $\mathrm{p}-\operatorname{LYN}(P=0.01)$ and $\mathrm{p}-\mathrm{PKC} \beta(P=0.01)$ were increased in stimulated risk and reference cells overexpressing LAG3 protein (lanes 2 and 3, respectively). As compared with mock-transduced reference cells, LAG3 silencing was associated with reduced p-LYN $(P=0.002)$ and $\mathrm{p}-\mathrm{PKC} \beta(P=0.009)$ (lane 6$)$. In lanes 4 and 5 , reference cells showed the expected increase in $\mathrm{p}$-LYN and $\mathrm{p}$-PKC $\beta$ following stimulation. The results were analyzed using a 2 -sided Student's $t$ test; $n=3, P<0.05$ was considered significant. 


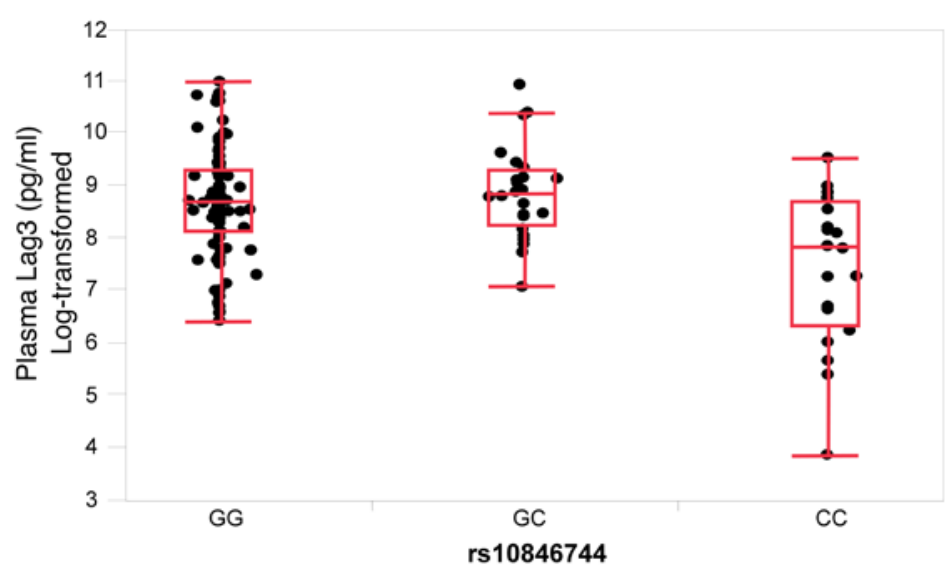

Figure 5. Association of SCARB1 rs10846744 genotypes with plasma LAG3 (log-transformed) in the HALP cohort. In the pooled analysis, homozygous carriers of the rs10846744 risk (CC) allele ( $n$ $=20$ ) had significantly lower plasma LAG3 levels compared with heterozygous $(n=24)$ and homozygous carriers of the (C) allele $(n=$ 102) (1-way ANOVA, $P<0.0001, n=146)$

( $n=633 ; 689[335 ; 1,920] \mathrm{pg} / \mathrm{ml} ; P<0.0001)$ compared with people of Mixed European Descent $(n=2,282 ; 425$ [209; $1,010] \mathrm{pg} / \mathrm{ml})$ and Hispanics $(n=1,275 ; 490$ [263; 1,131] pg/ ml) (Figure 8, plasma LAG3 shown as log-transformed). In a fully adjusted model, race $(P=0.0005)$, age $(P=0.003)$, lipid medications $(P=0.03)$, rs10846744 genotype $(P=0.002)$, and smoking status $(P<0.0001)$ were retained as independent predictors of plasma LAG3 levels (Table 2). Plasma LAG3 $(P$ $<0.007)$ remained an independent predictor of HDL-C levels even with adjustment by age $(P=0.006)$, sex $(P<0.0001)$, BMI $(P<0.0001)$, TG $(P<0.0001)$, alcohol use $(P<0.0001)$, hemoglobin $\mathrm{A} 1 \mathrm{c}\left(\mathrm{Hgb}_{\mathrm{Alc}}\right)(P=$ $0.01)$, and systolic $\mathrm{BP}(P=0.03)$ (Table 3$)$.

Logistic multivariable regression analysis revealed that plasma LAG3 was not significantly associated with subclinical atherosclerosis (common carotid intimal medial thickness [cIMT]) $(P=0.25)$ or coronary artery calcium score (CAC) $(P=0.062)$ (Supplemental Table 3). Logistic multivariable regression analysis revealed that, in addition to traditional risk factors such as age $(P<0.0001)$, sex $(P<0.0001)$, systolic $\mathrm{BP}(P$ $=0.0002), \operatorname{LDL}-\mathrm{C}(P=0.02), \mathrm{TC}(P=0.04)$, lipid medications $(P=0.002), \mathrm{Hgb}_{\mathrm{Alc}}(P=0.03)$, and smoking $(P=0.05)$, plasma LAG3 (beta -0.078 , odds ratio $[\mathrm{OR}] 1.15, P=0.02$ ) was an independent predictor of CHD (Table 4), but not for all-cause mortality.

The prevalence of participants within the MESA cohort with HDL-C greater than or equal to $60 \mathrm{mg} / \mathrm{dl}$ was $26 \%$, and within this group, the prevalence of CHD was $4 \%$ and not significantly different between the race/ ethnic groups (Supplemental Table 4). HDL-C levels in subjects with CHD ( $n=55,72.4 \pm 1.6 \mathrm{mg} / \mathrm{dl}$ ) were not significantly different compared with subjects without $\mathrm{CHD}(71.8 \pm 0.3 \mathrm{mg} / \mathrm{dl}, n=1,387, P=0.71)$. However, plasma LAG3 levels were 2-fold lower in subjects with CHD $(843.3 \pm 540.1 \mathrm{pg} / \mathrm{ml}, n=55)$ as compared with subjects without $\mathrm{CHD}(1,828 \pm 107.6 \mathrm{pg} / \mathrm{ml}, n=1,386, P=0.04)$. In a logistic regression analysis, plasma LAG3 (beta -0.212 , OR $1.45, P=0.004)$, age $(P=0.006)$, sex $(P=0.001)$, and diastolic BP $(P=0.03)$ were retained as independent predictors of $\mathrm{CHD}$ (Table 5).

We examined whether plasma LAG3 in the MESA cohort would significantly affect CHD risk prediction in comparison with the Framingham risk score (17). As shown in Table 6, plasma LAG3 increased CHD risk prediction in comparison with the Framingham risk score $(P=0.039)$. When the model included adjustment for study sites, race, and principal components (PCs) of ancestry, plasma LAG3 remained significant as a CHD risk predictor $(P=0.044)$

Given that we had observed a significant correlation between LAG3 and inflammatory markers from cultured B cells, we investigated whether plasma LAG3 was associated with inflammatory markers available in the MESA datasets. As shown in Table 7, following multivariable regression analysis plasma LAG3 was positively associated with IL-10 $(P<0.0001)$.

Figure 6. Association of race/ethnicity with plasma LAG3 (logtransformed) in the HALP cohort. African-Americans $(n=20)$ had significantly lower plasma LAC3 levels (log-transformed) compared with those of Mixed European Descent $(n=100)$, Hispanics $(n=6)$, and Asians $(n=7)$. One-way ANOVA, $P=0.004$, total $n=133$.

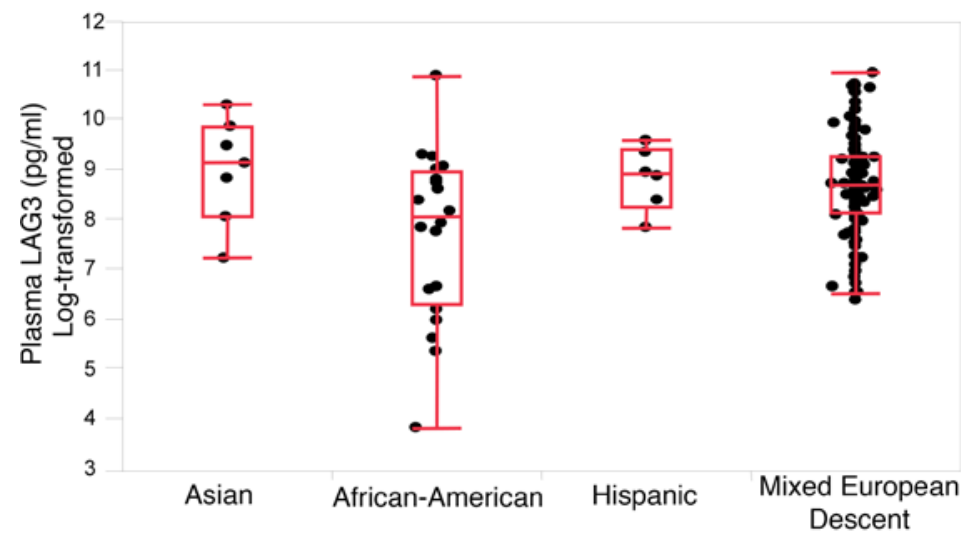


Table 2. Multivariable regression analysis of independent predictors for plasma LAG3: MESA

\begin{tabular}{|c|c|c|c|c|}
\hline Covariates $^{A}$ & $n$ & Beta & SEM & $P$ value \\
\hline \multicolumn{5}{|c|}{5,137} \\
\hline \multicolumn{5}{|l|}{ Rs10846744: } \\
\hline GG & & 183.98 & 91.1 & 0.04 \\
\hline GC & & -261.8 & 80.1 & 0.001 \\
\hline Race: & & & & 0.0005 \\
\hline Mixed European Descent & & -641.37 & 408.5 & \\
\hline Chinese & & 491.2 & $1,061.6$ & \\
\hline African American & & 862.7 & 450.2 & \\
\hline Age & & -19.81 & 6.7 & 0.003 \\
\hline Lipid meds & & 153.22 & 70.5 & 0.03 \\
\hline Smoking & & -10.95 & 2.72 & $<0.0001$ \\
\hline \multicolumn{5}{|c|}{$\begin{array}{l}\text { ACovariates included race, principle components of ancestry, age, study sites, sex, hemoglobin A1c, BMI, lipid } \\
\text { medications, lipids (total cholesterol, LDL-cholesterol, HDL-cholesterol), smoking pack years, systolic BP, and diastc } \\
\text { BP. LAG3 was log-transformed. GG, homozygous carriers of the rs10846744 reference genotype. GC, heterozygous } \\
\text { carriers of the rs10846744 reference allele (G) and the risk allele (C). }\end{array}$} \\
\hline
\end{tabular}

\section{Discussion}

The rationale for recruiting HALP subjects was to identify subjects with SCARB1 SNPs that were significantly associated with increased risk for atherosclerotic disease. We were able to identify significant associations of certain common SCARB1-coding SNPs with decreased expression and function of SR-BI protein (18). The rs10846744 SNP is significantly associated with atherosclerotic disease and it is a noncoding SNP residing in an enhancer region (6). Because the association of rs10846744 with CVD remained significant after multivariable regression analysis and in an effort to identify the pathway linking the association of rs10846744 with subclinical atherosclerosis and CVD, we used RNA-Seq and identified LAG3 RNA as a major target since its expression was significantly lower in homozygous carriers of the rs10846744 risk (CC) allele.

LAG3 protein deficiency altered phosphosignaling and enhanced a proinflammatory intracellular state as compared with reference-expressing (GG) cells (Figure 4). In the HALP subjects, we showed that plasma LAG3 protein levels were significantly lower in homozygous carriers of the rs 10846744 risk allele. When stratified by race/ethnicity, and regardless of genotyping, plasma LAG3 protein levels were lower in African-Americans as compared with Caucasian subjects. With multivariable regression adjusting for age, sex, and race, rs10846744 remained as an independent predictor of plasma LAG3 levels.

The LAG3 gene is located near the CD4 locus and LAG3 protein competes with CD4 by binding with higher affinity to MHC class II to negatively regulate T cell activation $(19,20)$. In murine cells, Kisielow et al. (16) reported that activated T cells induced LAG3 protein expression on B cells. In contrast, LAG3 RNA and LAG3 protein were detected in EBV-transformed B cells without coculture with T cells, with higher expression in EBV-transformed cells expressing the rs10846744 reference allele as compared with cells expressing the risk allele. Although EBV transformation of B lymphocytes could activate the cells, there was a significant difference in the level of LAG3 RNA and LAG3 protein expression based on rs10846744 genotype stratification.

Figure 7. Association of SCARB1 rs10846744 genotypes with plasma LAG3 (log-transformed) in MESA participants. In the pooled analysis, homozygous carriers of the risk rs10846744 (CC) allele ( $n$ = 974) had significantly higher plasma LAC3 levels compared with heterozygous $(n=2,185)$ and homozygous reference (GG) allele carriers $(n=2,448)$. One-way ANOVA,

$P<0.0001$, total $n=5,607$.

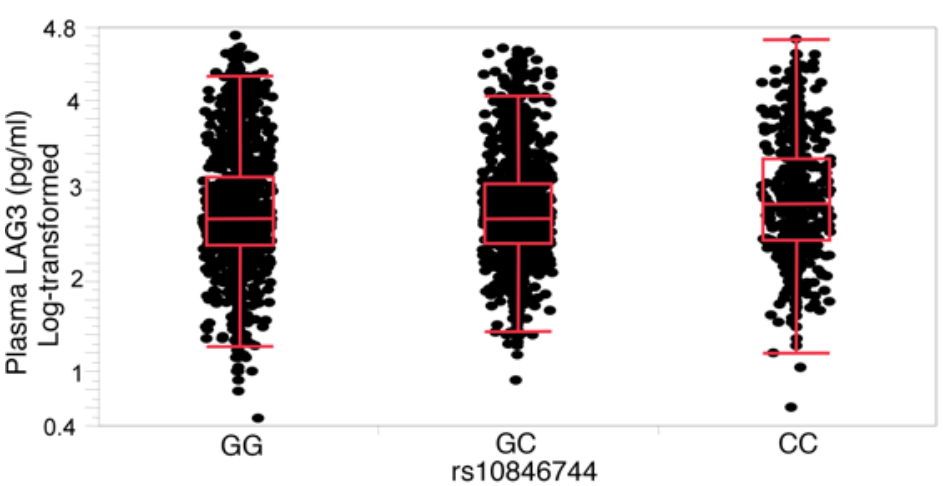


Table 3. Multivariable regression analysis of independent predictors for HDL-cholesterol: MESA

\begin{tabular}{|c|c|c|c|c|}
\hline Covariates $^{A}$ & $n$ & Beta & SEM & $P$ value \\
\hline \multicolumn{5}{|c|}{4,953} \\
\hline LAG3 & & -0.770 & 0.28 & 0.007 \\
\hline Age & & 0.056 & 0.02 & 0.006 \\
\hline Triglycerides & & -0.062 & 0.002 & $<0.0001$ \\
\hline Sex: Male & & 5.67 & 0.19 & $<0.0001$ \\
\hline BMI & & -0.623 & 0.035 & $<0.0001$ \\
\hline $\mathrm{Hgb}_{\mathrm{Alc}}$ & & -0.450 & 0.181 & 0.01 \\
\hline Alcohol & & 3.28 & 0.370 & $<0.0001$ \\
\hline Systolic BP & & 0.027 & 0.01 & 0.03 \\
\hline
\end{tabular}

Baixeras et al. (21) characterized the cellular distribution of LAG3 protein in a number of cell lines and demonstrated that LAG3 protein resided within lipid rafts. Subsequently, Woo et al. (22) reported the intracellular distribution of LAG3 protein as being equally distributed between intracellular compartments and the plasma membrane. By using flow cytometry, we confirmed that low levels of LAG3 protein were detected on the cell surface of risk-expressing cells regardless of stimulation conditions (Figure 2). However, LAG3 protein was expressed on the cell surface in unstimulated reference cells and levels decreased significantly after stimulation. In addition, since cell surface LAG3 is subject to cleavage by metalloproteases (13), we also observed significantly higher levels of soluble LAG3 protein in the culture media from reference-expressing cells as compared with the risk cells. Consistent with Huard et al. (9), we observed higher secretion levels of TNF- $\alpha$ from risk-expressing cells, demonstrating these cells exhibited a heightened state of inflammation.

LAG3 protein is one of a few important immune checkpoint inhibitors, including programmed cell death protein 1 (PD-1) and cytotoxic T lymphocyte antigen-4 (CTLA-4) (23-28). There has been a considerable amount of interest in PD-1 and CTLA-4 blockade, with biologicals such as pembrolizumab and ipilimumab approved by the FDA for clinical use for advanced melanoma (29-31). Snyder et al. (32) examined the genetic basis for clinical responsiveness to CTLA-4 blockade in subjects with malignant melanoma, finding that some subjects treated with CTLA-4 blockade derived minimal benefit. There is now a phase 1 clinical trial examining the safety and efficacy of anti-LAG3 monoclonal therapy alone and in combination with anti-PD1 in subjects with solid tumors (33). Our study now demonstrates a potentially novel connection between the SCARB1 rs10846744 SNP and LAG3 RNA and LAG3 protein, showing that carriers for this SNP have lower cellular expression of LAG3 protein and might not be as responsive to immunomodulatory therapy for malignancy.

$\mathrm{Bu}$ et al. (34) reported increased atherosclerosis and lesional activated CD4 ${ }^{+}$and $\mathrm{CD} 8^{+} \mathrm{T}$ cells in mice deficient in PD-1/PDL-1 and $L D L R^{-/-}$when fed a western diet as compared with control $L D L R^{+/+}$mice. Cochain et al. (35) observed similar findings as well as showing that a proinflammatory state predominates despite expansion of Tregs in PD-1-null mice. We now report that in MESA subjects plasma LAG3 was inversely associated with increased CHD but not with SCA or CAC. When MESA subjects were stratified by HDL greater than or equal to $60 \mathrm{mg} / \mathrm{dl}$, we observed a

Figure 8. Association of race/ethnicity with plasma LAG3 (logtransformed) in MESA participants. Chinese-Americans ( $n=633$ ) and African-Americans $(n=1,428)$ had significantly higher plasma LAC3 levels compared with people of Mixed European Descent ( $n=$ $2,282)$ and Hispanics $(n=1,275)(P<0.0001)$ and were not significantly different from each other. There were no significant differences in plasma LAG3 levels between patients of Mixed European Descent and Hispanics. One-way ANOVA, $P<0.0001$, total $n=561$.
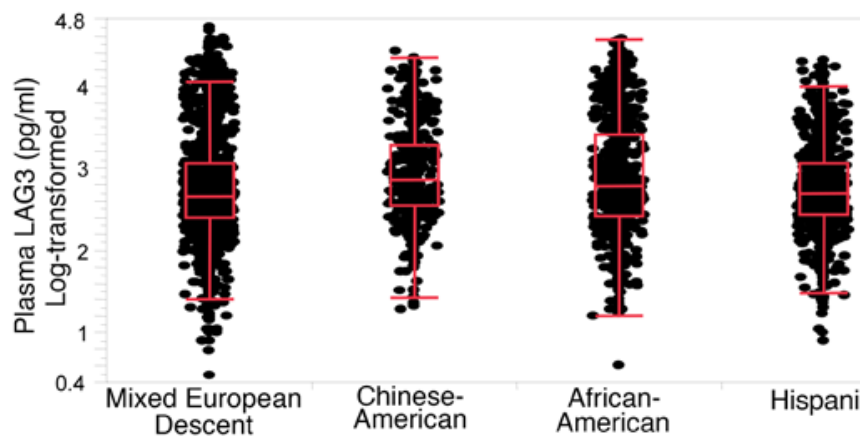

Descent 
Table 4. Association of plasma LAG3 with coronary heart disease in MESA participants

\begin{tabular}{|c|c|c|}
\hline Variable $^{A}$ & Odds Ratio & $95 \% \mathrm{Cl}$ \\
\hline LAG3 (log scale) ${ }^{\mathrm{B}}$ & 1.15 & $1.02-1.28$ \\
\hline $\operatorname{Age}^{\complement}$ & 2.41 & $1.81-3.21$ \\
\hline Sex & 2.10 & $1.50-2.94$ \\
\hline Systolic BPD & 1.60 & $1.25-2.06$ \\
\hline LDL-cholesterol $^{B}$ & 1.79 & $1.11-2.87$ \\
\hline Total cholesterol ${ }^{\mathrm{E}}$ & 0.60 & $0.37-0.97$ \\
\hline Lipid medication ${ }^{F}$ & 1.60 & $1.18-2.17$ \\
\hline $\mathrm{Hgb}_{\mathrm{A} 1 \mathrm{c}}$ & 1.09 & $1.01-1.14$ \\
\hline Smoking pack years ${ }^{\mathrm{H}}$ & 1.07 & $1.00-1.14$ \\
\hline \multicolumn{3}{|c|}{$\begin{array}{l}{ }^{\mathrm{A} C o v a r i a t e s} \text { included race, principle components of ancestry, age, study sites, sex, hemoglobin } \mathrm{A} 1 \mathrm{c}(\mathrm{Hgb} \mathrm{Alc}), \mathrm{BMI}, \\
\text { lipid medications, lipids (total cholesterol and LDL-cholesterol), smoking pack years, systolic BP, and diastolic BP. } \\
\text { The estimated coefficient of LAC3 was }-0.078 \text { (SEM } 0.034 \text { ). For LAC3, the odds ratio was estimated by comparing } \\
\text { the first quartile vs. the third quartile of the variables. For the other continuous variables, the odds ratios were } \\
\text { estimated by comparing third quartile vs. first quartile of the variables. }{ }^{\mathrm{B}} P=0.02 ;{ }^{\mathrm{C}} n=4707 \text { and } P<0.0001 ;{ }^{\mathrm{D}} P= \\
0.0002 ;{ }^{\mathrm{E}} P=0.04 ;{ }^{\mathrm{F}} P=0.002 ;{ }^{\mathrm{G}} P=0.03 ;{ }^{\mathrm{H}} P=0.05\end{array}$} \\
\hline
\end{tabular}

prevalence of HALP within the entire MESA population at $26 \%$. We did not observe a significant difference between HDL-C levels in subjects with or without CHD, but did observe 2-fold lower plasma LAG3 levels in MESA subjects with HALP and CHD compared with subjects without CHD. Consistent with what was observed in the in vitro experiments (Figure 3B), low plasma LAG3 levels were significantly associated with low circulating levels of IL-10 in the MESA participants. A connection between LAG3 and IL-10 has been shown by Gagliani et al. (36), by which these investigators identified cell surface markers CD49b and LAG3 as markers of Treg type I cells that secrete IL-10. Thus, it seems plausible that low plasma LAG3 is significantly associated with low plasma IL-10.

The HALP cohort is a unique population of healthy subjects recruited based on high HDL-C levels ( $\geq 60 \mathrm{mg} / \mathrm{dl}$ ) (4). Fasting plasma samples were obtained at 1 visit and thus the data are representative of a cross-sectional study, as is true for the MESA samples. The results from the in vitro studies were obtained from EBV-transformed B cells.

In summary, our study shows a potentially novel connection between rs10846744 and LAG3 RNA and LAG3 protein expression and LAG3 with HDL and CHD, supporting further studies examining the intersection of this important immune checkpoint inhibitor on T cell activation, HDL metabolism, and increased risk for CVD. Moreover, plasma LAG3 levels could be informative in the risk stratification for patients with HALP, as it is now becoming clear that current pharmacologic efforts to raise HDL-C levels have not led to improved CVD outcomes (37-39). It is compelling that plasma LAG3 levels might be an excellent biomarker for risk stratification in subjects with HALP, especially since we found that plasma LAG3 provided additional information about CHD risk prediction compared with the Framingham risk score.

Table 5. Association of plasma LAG3 with coronary heart disease in MESA participants with HDLcholesterol $\geq 60 \mathrm{mg} / \mathrm{dl}$

\begin{tabular}{|c|c|c|}
\hline Variable $^{A}$ & Odds Ratio & $95 \% \mathrm{Cl}$ \\
\hline LAG3 (log scale) ${ }^{\mathrm{B}}$ & 1.45 & $1.12-1.85$ \\
\hline $\mathrm{Age}^{\mathrm{c}}$ & 2.79 & $1.35-5.78$ \\
\hline $\operatorname{Sex}^{D}$ & 3.80 & $1.68-8.56$ \\
\hline Diastolic BPE & 0.49 & $0.26-0.92$ \\
\hline
\end{tabular}

ACovariates included race, principle components of ancestry, age, study sites, sex, hemoglobin A1c, BMI, lipid medications, lipids (total cholesterol and LDL-cholesterol), smoking pack years, systolic BP, and diastolic BP. The estimated coefficient of LAC3 was -0.212 (SEM 0.073). For LAG3, the odds ratio was estimated by comparing the first quartile vs. the third quartile of the variables. For the other continuous variables, the odds ratios were estimated by comparing the third quartile vs. the first quartile of the variables. ${ }^{\mathrm{B}} n=1,134$ and $P=0.004 ;{ }^{\mathrm{C}} P=0.006 ;{ }^{\mathrm{D}} P=0.001 ;{ }^{\mathrm{E}} P=0.03$. 
Table 6. Plasma LAG3 as a coronary heart disease (CHD) risk predictor compared with Framingham risk score

\begin{tabular}{|c|c|c|c|}
\hline & Beta & SEM & $P$ value \\
\hline \multicolumn{4}{|l|}{ Model 1: $(n=5,468)$} \\
\hline Framingham risk score (log scale) & 0.872 & 0.093 & $<0.0001$ \\
\hline \multicolumn{4}{|l|}{ Model 2: $(n=5,468)$} \\
\hline Framingham risk score (log scale) & 0.875 & 0.094 & $<0.0001$ \\
\hline LAG3 (log scale) & -0.065 & 0.031 & 0.039 \\
\hline \multicolumn{4}{|l|}{ Model 3: $(n=5,468)$} \\
\hline Framingham risk score (log scale) & 0.891 & 0.095 & $<0.0001$ \\
\hline \multicolumn{4}{|l|}{ Model 4: $(n=5,468)$} \\
\hline Framingham risk score (log scale) & 0.893 & 0.095 & $<0.0001$ \\
\hline LAG3 (log scale) & -0.064 & 0.031 & 0.044 \\
\hline \multicolumn{4}{|c|}{$\begin{array}{l}\text { Likelihood ratio test of comparing model } 2 \text { (log-transformed plasma LAG3) with model } 1 \text { (Framingham risk score; see } \\
\text { ref. 17) indicated that inclusion of plasma LAC3 provided significant additional information in predicting CHD risk }(P= \\
0.039) \text {. Models } 3 \text { and } 4 \text { were adjusted for study sites, race, and PCs of ancestry; the likelihood ratio test of comparing } \\
\text { model } 4 \text { with model } 3 \text { confirmed that inclusion of LAG3 provided significant additional information in predicting CHD } \\
\text { risk }(P=0.044) \text {. }\end{array}$} \\
\hline
\end{tabular}

\section{Methods}

\section{Study subjects}

HALP participants. Community-dwelling adults between the ages of 18 and 81 years with fasting plasma HDL-C levels greater than or equal to $60 \mathrm{mg} / \mathrm{dl}$ (HALP) were enrolled in this ongoing clinical study (4). The rationale for recruiting HALP subjects was to identify subjects with SCARB1 variants causally linked to increased atherosclerotic risk, which we have now shown with 2 variants, rs 4238001 and rs10846744 $(6,18)$. Subjects consented to provide overnight fasting blood samples for analysis of lipid profile, DNA analysis for SCARB1 genotyping, and lymphocyte isolation from buffy coats (4).

MESA participants. The rationale for replicating plasma LAG3 associations in the MESA cohort was the fact that MESA is a large, racially/ethnically diverse study of the natural history of atherosclerotic disease in adults with some collection of risk factors and biomarkers that are identical to those in the HALP study (lipid measurements, rs10846744 genotyping, and plasma LAG3 levels), while having endpoints that are not available in HALP (cIMT, CAC, CHD outcomes, and all-cause mortality) (40). Between 2000 and 2002, MESA recruited 6,814 men and women 45-84 years of age from Forsyth County, North Carolina; New York City, New York; Baltimore, Maryland; St. Paul, Minnesota; Chicago, Illinois; and Los Angeles. The formal adjudications for confirmed CHD (described by MESA as CHD-hard) and all-cause mortality have been previously described (6). The replication design was based on availability of frozen fasting plasma samples from a single visit (Exam 2). The longitudinal morbidity and mortality data were obtained from recorded events from visit 2 (2002-2004) to visit 5 (2013).

Table 7. Association of plasma LAG3 with inflammatory markers in MESA participants

\begin{tabular}{lcccc}
\hline Outcomes & $\boldsymbol{n}$ & Beta & SEM & \multicolumn{1}{c}{$\boldsymbol{P}$ value } \\
IL-2 & 2,386 & -0.002 & 0.004 & 0.624 \\
IL-6 & 5,414 & -0.006 & 0.044 & 0.203 \\
IL-10 & 2,350 & 0.035 & 0.007 & $<0.0001$ \\
CD40 ligand & 851 & -0.0004 & 0.010 & 0.966 \\
sTNF $\alpha$ R & 2,396 & -0.001 & 0.003 & 0.824 \\
hs-CRP & 5,533 & -0.013 & 0.007 & 0.087
\end{tabular}

Regression models were adjusted for age, sex, study site, race, and principal components of ancestry. All outcome variables and plasma LAG3 were log transformed. sTNF $\alpha$ R, soluble TNF- $\alpha$ receptor; hs-CPR, high-sensitivity C-reactive protein. 
Materials and experimental assays

Details are provided in the Supplemental file.

\section{Genetic analysis in MESA}

Genotyping for rs10846744 in MESA was completed on the CardioMetabochip, a custom genotyping array composed of SNPs selected to be associated with type 2 diabetes mellitus and obesity-related traits. PCs of ancestry were computed within race/ethnic groups using the full set of CardioMetabochip genotypes using SMARTPCA (41).

\section{Statistics}

One-way ANOVA was used for testing group differences, and 2-sided Student's $t$ test was used for 2-sample analysis. Quadratic polynomial regression analyses were performed to allow for a nonlinear relationship with time. A $P$ value less than 0.05 was considered significant. Linear regression analyses in HALP were performed for the log-transformed outcomes of LAG3 levels within race/ethnic groups, each covariate adjustment for age and sex, and with additional adjustment for race/ethnic group in pooled analysis. In MESA, linear regression analyses with pooled samples used a fully adjusted model for race, PCs of ancestry, age, sex, study site, $\mathrm{Hgb}_{\mathrm{Alc}}$, BMI, lipids (TC, LDL-C, HDL-C, TG), lipid-lowering medications, systolic and diastolic BP, pack years of smoking and, in some analyses, rs 10846744 genotype and alcohol use.

\section{Study approval}

For HALP participants, subjects provided written informed consent prior to their participation in the study, and this study was approved by the institutional review boards at the Johns Hopkins University School of Medicine, Baltimore, Maryland, USA and the University of Connecticut School of Medicine. For MESA participants, study subjects provided written informed consent prior to their participation in the study and the MESA study was approved by the institutional review boards at each participating institution, including the Johns Hopkins University School of Medicine Office of Human Subjects Research Institutional Review Boards; University of Minnesota Human Research Protection Program, Minneapolis, Minnesota, USA; University of California Los Angeles Office of the Human Research Protection Program; Northwestern University Institutional Review Board Office, Chicago, Illinois, USA; Wake Forest University Health Sciences Office of Research Institutional Review Board, Winston-Salem, North Carolina, USA; and Columbia University Medical Center Institutional Review Board, New York, New York, USA.

\section{Author contributions}

DG helped design the research study, conducted experiments, acquired and analyzed data, and wrote the manuscript. AK helped design the research study and acquired and analyzed data. SS helped design the research study, conducted experiments, and acquired and analyzed data. ATV helped analyze the data and wrote the manuscript. AM helped acquire and analyze data and wrote the manuscript. XQ Wang analyzed data. SJB, KDT, and YDIC acquired data. SSR acquired and analyzed data and wrote the manuscript. AR helped design the research study, acquired and analyzed data, and wrote the manuscript.

\section{Acknowledgments}

We would like to acknowledge the assistance from the staff of the Lowell P. Weicker, Jr. Clinical Research Center at the University of Connecticut Health Center as well as the staff from the Johns Hopkins Bayview Medical Center Clinical Research Unit for help in recruiting the HALP cohort. Although the research described in this manuscript has been funded in part by the United States Environmental Protection Agency through grant RD831697 to the University of Washington, it has not been subjected to the Agency's required peer and policy review and therefore does not necessarily reflect the views of the Agency and no official endorsement should be inferred.

Address correspondence to: Annabelle Rodriguez, Professor of Cell Biology, Linda and David Roth Chair of Cardiovascular Research, University of Connecticut Health Center, L Building, Room 5021, 263 Farmington Avenue, Farmington, Connecticut 06030, USA. Phone: 860.679.2463; E-mail: rodriguezoquendo@uchc.edu. 
1. Acton S, Rigotti A, Landschulz KT, Xu S, Hobbs HH, Krieger M. Identification of scavenger receptor SR-BI as a high density lipoprotein receptor. Science. 1996;271(5248):518-520.

2. Rigotti A, Trigatti BL, Penman M, Rayburn H, Herz J, Krieger M. A targeted mutation in the murine gene encoding the high density lipoprotein (HDL) receptor scavenger receptor class B type I reveals its key role in HDL metabolism. Proc Natl Acad Sci US A. 1997;94(23):12610-12615.

3. Trigatti B, et al. Influence of the high density lipoprotein receptor SR-BI on reproductive and cardiovascular pathophysiology. Proc Natl Acad Sci U S A. 1999;96(16):9322-9327.

4. West M, et al. Scavenger receptor class B type I protein as an independent predictor of high-density lipoprotein cholesterol levels in subjects with hyperalphalipoproteinemia. J Clin Endocrinol Metab. 2009;94(4):1451-1457.

5. Naj AC, et al. Association of scavenger receptor class B type I polymorphisms with subclinical atherosclerosis: the Multi-Ethnic Study of Atherosclerosis. Circ Cardiovasc Genet. 2010;3(1):47-52.

6. Manichaikul A, Naj AC, Herrington D, Post W, Rich SS, Rodriguez A. Association of SCARB1 variants with subclinical atherosclerosis and incident cardiovascular disease: the multi-ethnic study of atherosclerosis. Arterioscler Thromb Vasc Biol. 2012;32(8):1991-1999.

7. Zanoni P, et al. Rare variant in scavenger receptor BI raises HDL cholesterol and increases risk of coronary heart disease. Science. 2016;351(6278):1166-1171.

8. The ENCODE Project: ENCyclopedia Of DNA Elements. US National Human Genome Research Institute. http://genome. ucsc.edu. Updated March 31, 2015. Accessed September 21, 2016.

9. Huard B, Tournier M, Hercend T, Triebel F, Faure F. Lymphocyte-activation gene 3/major histocompatibility complex class II interaction modulates the antigenic response of CD4+ $\mathrm{T}$ lymphocytes. Eur J Immunol. 1994;24(12):3216-3221.

10. Triebel F. LAG-3: a regulator of T-cell and DC responses and its use in therapeutic vaccination. Trends Immunol. 2003;24(12):619-622.

11. Workman CJ, et al. LAG-3 regulates plasmacytoid dendritic cell homeostasis. J Immunol. 2009;182(4):1885-1891.

12. Workman CJ, Vignali DA. The CD4-related molecule, LAG-3 (CD223), regulates the expansion of activated T cells. Eur J Immunol. 2003;33(4):970-979.

13. Li N, et al. Metalloproteases regulate T-cell proliferation and effort function via LAG-3. EMBO J. 2007;26(2):494-504.

14. Lienhardt C, et al. Active tuberculosis in Africa is associated with reduced Th1 and increased Th2 activity in vivo. Eur J Immunol. 2002;32(6):1605-1613.

15. Triebel F, Hacene K, Pichon M-F. A soluble lymphocyte activation gene-3 (sLAG-3) protein as a prognostic factor in human breast cancer expressing estrogen or progesterone receptors. Cancer Letters. 2006;235(1):147-153.

16. Kisielow M, Kisielow J, Capoferri-Sollami G, Karjalainen K. Expression of lymphocyte activation gene 3 (LAG-3) on B cells is induced by T cells. Eur J Immunol. 2005;35(7):2081-2088.

17. Wilson PW, D'Agostino RB, Levy D, Belanger AM, Silbershatz H, Kannel WB. Prediction of coronary heart disease using risk factor categories. Circulation. 1998;97(18):1837-1847.

18. Manichaikul A, et al. Association of the lipoprotein receptor SCARB1 common missense variant rs4238001 with incident coronary heart disease. PLoS One. 2015;10(5):e0125497.

19. Hannier S, Triebel F. The MHC class II ligand lymphocyte activation gene-3 is co-distributed with CD8 and CD3-TCR molecules after their engagement by mAb or peptide-MHC class I complexes. Int Immunol. 1999;11(11):1745-1752.

20. Sierro S, Romero P, Speiser DE. The CD4-like molecule LAG-3, biology and therapeutic applications. Expert Opin Ther Targets. 2011;15(1):91-101.

21. Baixeras E, et al. Characterization of the lymphocyte activation gene 3-encoded protein. A new ligand for human leukocyte antigen class II antigens. J Exp Med. 1992;176(2):327-337.

22. Woo SR, et al. Differential subcellular localization of the regulatory T-cell protein LAG-3 and the coreceptor CD4. Eur J Immunol. 2010;40(6):1768-1777.

23. Nguyen LT, Ohashi PS. Clinical blockade of PD1 and LAG3-potential mechanisms of action. Nat Rev Immunol. 2015;15(1):45-56.

24. Walunas TL, et al. CTLA-4 can function as a negative regulator of T cell activation. Immunity. 1994;1(5):405-413.

25. Keir ME, Butte MJ, Freeman GJ, Sharpe AH. PD-1 and its ligands in tolerance and immunity. Annu Rev Immunol. 2008;26:677-704.

26. Berman D, Korman A, Peck R, Feltquate D, Lonberg N, Canetta R. The development of immunomodulatory monoclonal antibodies as a new therapeutic modality for cancer: the Bristol-Myers Squibb experience. Pharmacol Ther. 2015;148:132-153.

27. Tsai KK, Zarzoso I, Daud AI. PD-1 and PD-L1 antibodies for melanoma. Hum Vaccin Immunother. 2014;10(11):3111-3116.

28. Tumeh PC, et al. PD-1 blockade induces responses by inhibiting adaptive immune resistance. Nature. 2014;515(7528):568-571.

29. Poole RM. Pembrolizumab: first global approval. Drugs. 2014;74(16):1973-1981.

30. Robert C, et al. Anti-programmed-death-receptor-1 treatment with pembrolizumab in ipilimumab-refractory advanced melanoma: a randomized dose-comparison cohort of a phase 1 trial. Lancet. 2014;384(9948):1109-1117.

31. Hamid O, et al. Safety and tumor responses with lambrolizumab (anti-PD-1) in melanoma. N Engl J Med. 2013;369(2):134-144.

32. Snyder A, Makarov V, Merghoub T, Yuan J, Zaretsky JM, Desrichard A, et al. Genetic basis for clinical response to CTLA-4 blockade in melanoma. N Engl J Med. 2014;371(23):2189-2199.

33. Bristol-Myers Squibb. Safety study of anti-LAG-3 with and without anti-PD-1 in the treatment of solid tumors. Clinical Trials. gov. https://clinicaltrials.gov/ct2/show/NCT01968109. Updated August 23, 2016. Accessed September 21, 2016.

34. Bu DX, et al. Impairment of the programmed cell death-1 pathway increases atherosclerotic lesion development and inflammation. Arterioscler Thromb Vasc Biol. 2011;31(5):1100-1107.

35. Cochain C, Chaudhari SM, Koch M, Wiendl H, Eckstein HH, Zernecke A. Programmed cell death-1 deficiency exacerbates $\mathrm{T}$ cell activation and atherogenesis despite expansion of regulatory T cells in atherosclerosis-prone mice. PLoS One. 2014;9(4):e93280.

36. Gagliani N, et al. Coexpression of CD49b and LAG-3 identifies human and mouse T regulatory type 1 cells. Nat Med. 2013;19(6):739-746

37. Schwartz GG, et al. Effects of dalcetrapib in patients with a recent acute coronary syndrome. N Engl J Med. 2012;367(22):2089-2099.

38. Barter PJ, et al. Effects of torcetrapib in patients at high risk for coronary events. N Engl J Med. 2007;357(21):2109-2122. 
39. AIM-HIGH Investigators, et al. Niacin in patients with low HDL cholesterol levels receiving intensive statin therapy. $N$ Engl $J$ Med. 2011;365(24):2255-2267.

40. Bild DE, et al. Multi-Ethnic Study of Atherosclerosis: objectives and design. Am J Epidemiol. 2002;156(9):871-881.

41. Price AL, Patterson NJ, Plenge RM, Weinblatt ME, Shadick NA, Reich D. Principal component analysis corrects for stratification in genome-wide association studies. Nat Genet. 2006;38(8):904-909. 